\title{
On-line Monitoring of SRM Grain Structure Based on embedded FBG Sensor
}

\author{
ZHANG Lei, CHANG Xin-Long, ZHANG You-hong, Chen Xiang-dong, ZHANG Qing
}

Xi'an Hi-Tech Institute, Xi'an, Shanxi 710025, China

\begin{abstract}
This paper proposes the use of FBG (Fiber Bragg grating) sensors for SRM (Solid Rocket Motor) grain structure continuous Structural Health Monitoring(SHM) as they are generally considered as the preferable solution in flammable and explosive environments, due to their electromagnetic interference immunity and their inherent electrical and explosion safe characteristics. The processes of SRM manufacturing(curing of propellant and demoulding ) were monitored. The curing degree of the propellant can be monitored by wavelength change of the FBG sensors, and this provides a new monitoring method for curing of the propellant grain. The monitoring of the demoulding process shows that the sensor is very sensitive to the tensile and compressive stresses on grain and can be used for on-line monitoring of grain in a mechanical environment. In monitoring simulated debonding test, the failure process of crack initiation, expansion and direction all can be monitored by the FBG sensors. After the crack is produced, the location of the debonding can be determined by the variation of the different sensors.
\end{abstract}

\section{Introduction}

Rocket motors are one of the largest items that dictate missile system service life. Thus, structure health monitoring is of significant interest for the safety and reliability of solid rocket motors (SRMs)[1]. The ability to detect damage in SRMs will help enable timely, accurate, and reliable assessment of structural integrity, and thus will yield great cost savings and prevent catastrophic structural failures. Classical approaches used to predict and detect material degradation have been to develop aging models for predicting the state of a material, given an assumed or measured environmental history, and the use of non-destructive testing methods such as ultrasound and X-rays. Both approaches, as currently practiced, are inadequate to meet the needs of a real-time, self-sensing health monitoring system. Thus, in recent years efforts have been devoted to investigate an entirely new approach to meet the goal of self-sensing ordnance the use of embedded sensors[1]. Several types of embedded sensors are being investigated in the solid rocket motor community[2]. Bond line sensors are small pressure sensors used to measure the stress between the propellant and case. The sensors are used to detect the perturbation in the stress field due to the presence of damage. Among the several types of sensors that could be considered, electrical strain-gage-based bond stress sensors, which measure the normal stress between the grain and liner, have received the most attention, and it has been shown that they can have significant value in their ability to validate grain stress predictions $[3,4]$. However, the need for electrical leads entering the motor presents safety concerns that may preclude this method except for laboratory applications. Optical fiber strain sensors, particularly Fiber Bragg grating (FBG) strain sensors, have also received some attention, optical sensors are considered as the preferable solution in such environments, due to their electromagnetic interference immunity and their inherent electrical and explosion safe characteristics. Furthermore, FBG are ideal for multiple sensor applications as many of these gratings can be employed either in series or in parallel. Udd and Benterou[5]used optical fiber Bragg grating sensors to measure velocity, position, temperature, and pressure in the interior of motors, and similar technologies have also been used to detect impact damage to the case material. Riziotis et al[6]. further applied a strain-sensing technology using photonic polymer optical fibers for local-deformation monitoring of propellant grains. Lopatin and Grinstein [7] recently introduced an active sensing method, by combining the magnetic induction for excitation and optical fiber Bragg grating sensors, for monitoring the integrity of solid propellant without implementing electrical wires into the rocket motor. These fiber Bragg technologies have been demonstrated as a means to study grain integrity. However, several issues with fiber optics that need to be addressed and overcome before this technology can be used in general in SRMS. Fibers are typically made from brittle material such as glass. This results in being fragile and difficult to handle. This paper proposes a new method using fiber Bragg gratings by polymer packaging sensors for measuring the bond stress between the propellant and insulation. There are three advantages for using polymer 
packaging the FBG. Firstly, the FBG is so very brittle that the survival rate is difficult to guarantee in the embedding process. It needs special protection to prevent breaking. Secondly, the bubbles or impurities may be formed around the FBG in embedding process. They will generate stress concentration causing the spectrum peak splitting or chirp which provides an unambiguous indication of the strain, and it is difficult to interpret the results. Lastly, the bond stress is perpendicular the bond interface embedded FBG sensor. The lateral pressure tuning of FBGs suffers from the drawback of low lateral pressure sensitivity, narrow tuning range and stress induced birefringence. This can be achieved by coating or packaging the FBG with a material having low elastic modulus and very high Poisson's ratio, which can effectively transfer the stress applied in one direction to the mutually perpendicular directions. In this paper, the FBG sensors were coated by wafers' package of HTPB/TDI elastic mass.

\section{Monitoring Process of SRM Manufacturing}
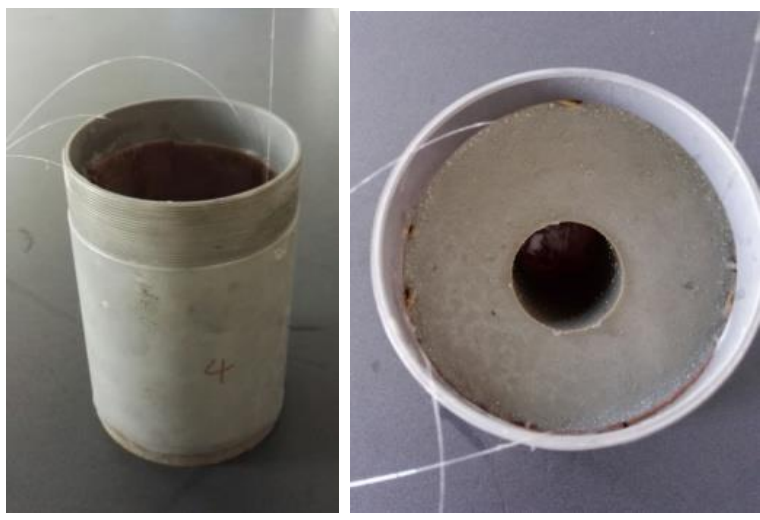

Figure 1. Analogue motor

The analogue motor geometry is shown in Figure 1. The inner and outer grain diameters are $27 \mathrm{~mm}$ and $94 \mathrm{~mm}$, respectively. The thicknesses of the insulation and case are $2.5 \mathrm{~mm}$ and $3 \mathrm{~mm}$, respectively. The propellant is a typical composite grain of HTPB/ammonium perchlorate (HTPB/AP). The insulation layer is ethylene propylene diene monomer (EPDM). The motor case is made of steel. There were eight sensors arrangement on the inside of the motor tubes. The sensors S1, S2, S3, S4 were arranged in the way of hoop and $\mathrm{H} 1, \mathrm{H} 2$ were arranged in the way of axial. The arrangement of the sensor is shown in Figure 2. The motor was monitored during the curing process. The sm130 Optical sensing module of MOI Company was used in the experiment.
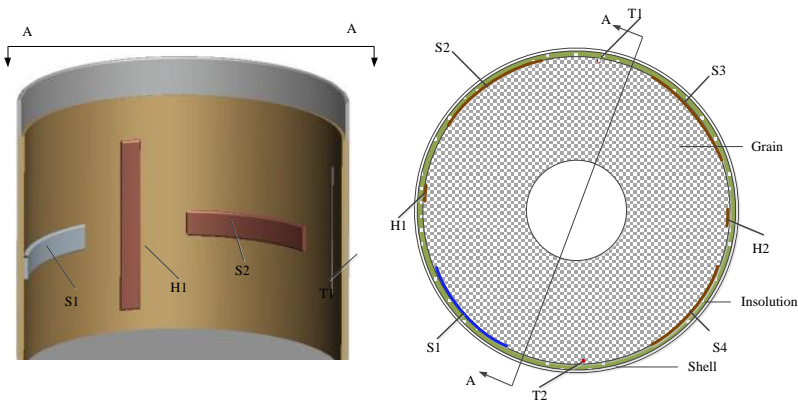

Figure 2. Schematic distribution of sensors

\subsection{Monitoring Curing of Simulated Motor}

The curing temperature is $70^{\circ} \mathrm{C}$. As shown in Figure 3, the change of the wavelength of the motor during the curing process under the constant temperature conditions is shown. It can be seen from the figure that the wavelength of the entire curing process decreases with the increase of the curing time, which means that the sensor is affected by the curing process. The tensile stress, the sensor's final wavelength changes more and more slowly as curing progresses, and the sensor's center wavelength eventually stabilizes at a fixed value. In the actual propellant manufacturing process, the positive curing point of the propellant needs to be determined. The positive curing point refers to the starting time when the propellant slurry is basically completed at a certain temperature and the mechanical properties do not change with the heating time. The method for determining the positive curing point in the project is to randomly cast 2 to 3 billets and cure them together with the motor during the charging and pouring process. After a predetermined constant temperature for several hours, take a billet at regular intervals to test its mechanical properties at room temperature until the maximum elongation at the test is close to the elongation at break and the tensile strength reaches the predetermined value at the same time. Has reached the curing point. It can be seen that determining the positive curing point through the billet test piece not only has a complicated process, but also often has uncertainty in the mechanical properties of the propellant and the change of the wavelength of the FBG sensor can effectively reflect the curing degree of the propellant grain. Provides a new method for curing monitoring of the grain.

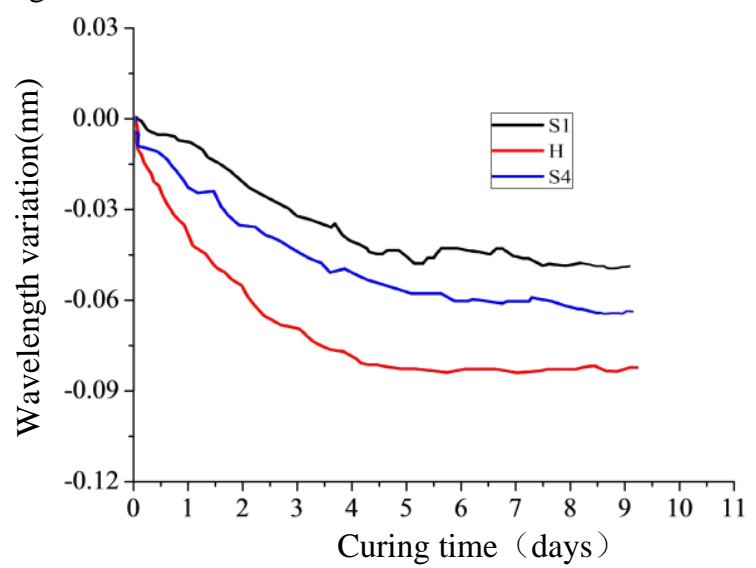

Figure 3. Wavelength variation of FBG sensor during Curing 


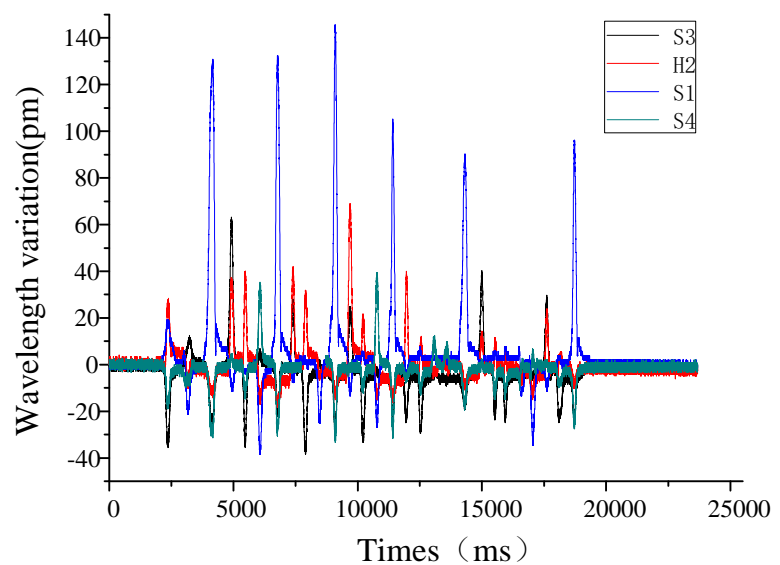

Figure 4. Sensor response during demolding

\subsection{Monitoring of Demoulding Process}

As shown in Figure 4, the relationship between wavelength change and time during demolding. During the demoulding process, the local pressure of the sensor is increased or decreased due to the shaking of the mandrel. The change of the wavelength of the sensor in the demolding process can be clearly seen from the figure. When the wavelength increases, it is subjected to a pressure effect. When the wavelength is reduced, it is subjected to tensile stress. A small increase in sensor wavelength occurs after the core has been withdrawn, indicating that there is a certain amount of adhesion stress between the core and the propellant. It can be seen that the sensor is very sensitive to the tensile and compressive stress on the drug column and can be used to monitor the stress and strain conditions of the drug column under mechanical conditions.

\subsection{Monitoring Simulated Debonding Test}

To study the stress response of the sensor during the debonding process, a debonding defect was artificially created using a blade, as shown in Figure 5 the positional relationship between the sensor and the debonding area. The entire process of manufacturing defects is recorded using the sm130. The frequency of $1000 \mathrm{~Hz}$ is used to set the relationship between the wavelength variation and time, as shown in Figure 6. Among them, the crack extends from the $\mathrm{S} 1$ position to the $\mathrm{S} 4$ position in the figure, with a deboding depth of $20 \mathrm{~mm}$, length of 10 $\mathrm{mm}$, and a crack debonding area of $150 \mathrm{~mm}$. When the blade is used for debonding, the cracking direction of the debonding crack is from the $\mathrm{S} 1$ position to the $\mathrm{S} 4$ position, and the force applied to the two sensors is reversed. The S4 sensor area is squeezed, so that the sensor pressure value increases, the sensor center wavelength increases, and the S1 sensor area is subjected to a tensile stress as the blade moves after inserting the blade, and thus the center wavelength may decrease. It can also be seen from the figure that since the debonding site is above the sensor $\mathrm{H} 2$, its wavelength variation is the largest. When the blade is withdrawn, the other sensor wavelengths are almost returned to the initial state, and the sensor $\mathrm{H} 2$ is obviously increased. This is due to debonding. As a result, the tensile stress it receives is reduced, resulting in an increase in its wavelength. It can be seen that the sensor can fully record the stress changes during the debonding process, and proves that the sensor and the monitoring system have strong sensitivity. The failure process of crack initiation, expansion and direction all can be monitored by the FBG sensors. After the crack is produced, the location of the debonding can be determined by the variation of the different sensors. Changes in bond stress after de-bonding can only be evident on sensor test curves near the defect, while other sensors do not change significantly.

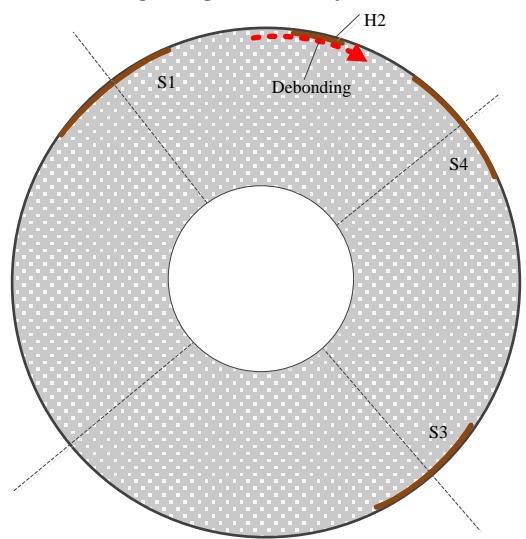

Figure 5. Sensor and location of debonding

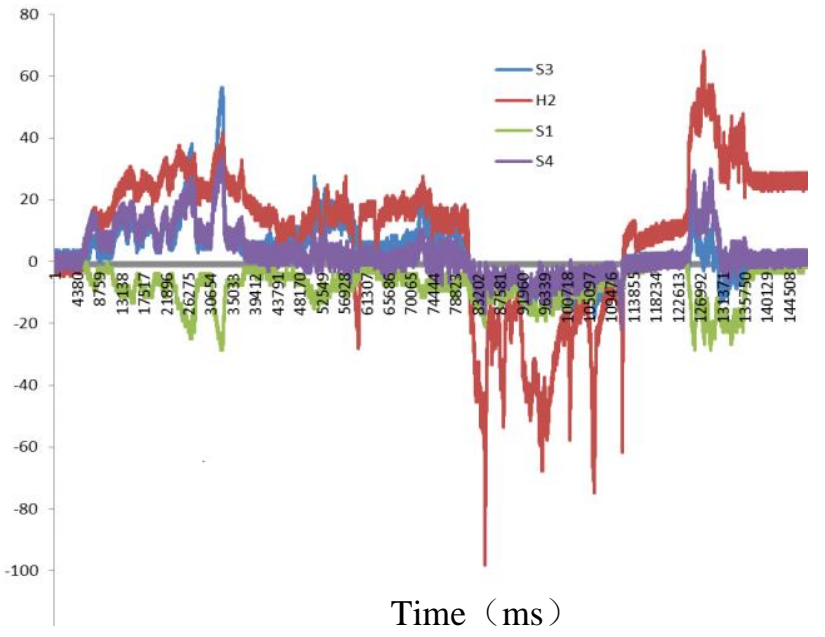

Figure 6. Sensors response curve in process of debonding

\section{Conclusion}

The embedded sensors can monitor the curing degree of the propellant slurry through the wavelength change of the FBG sensor, and provide a new monitoring method for curing of the propellant grain. During the demoulding process, the pressure of the sensor is increased or decreased due to shaking of the mandrel, and the force of the grain can be monitored through the change of the wavelength of the sensor. The monitoring of the demoulding process shows that the sensor is very sensitive to the tensile and compressive stresses on grain and can be used for on-line monitoring of grain in a mechanical environment. Sensors on both sides of the debonding area show different viration of wavelength trends in the crack initiation process, which is due to the crack initiation direction. The sensor near the beginning 
of the crack is subjected to tensile stress and its center wavelength decreases. The sensor near the terminating end is subjected to compressive stress and its center wavelength increases. The sensor that falls into the debonding area has the largest wavelength variation, and the change in the bond stress after the debonding can be clearly reflected on the sensor test curve near the defect, while other sensors do not change significantly.

\section{References}

1. Little, R. R., Chelner, H., and Buswell, H. J. (2006). "Development, testing, and application of embedded sensors for solid rocket motor health monitoring." Proc., 37th Annual Conf. of Fraunhofer Institute for Chemical Technology (ICT), Fraunhofer Institute for Chemical Technology, Pfintzal, Germany.

2. Craig M. Lopatin : U.S. Patent US 7652488 B1 (2010).

3. Anhduong Q. Le, A.M.ASCE1, L. Z. Sun, Ph.D., M.ASCE2; and Timothy C. Miller, Ph.D."Health
Monitoring and Diagnosis of Solid Rocket Motors with Bore Cracks" Journal of Aerospace Motorering, ASCE, 2016, 29(3): 04015058 .

4. Le, A. Q., Sun, L. Z., and Miller, T. C. (2013). "Detectability of delaminations in solid rocket motors with embedded stress sensors." J. Propul. Power, 29(2),299-304.

5. Riziotis, C., Eineder, L., Bancallari, L., and Tussiwand, G. (2013). "Fiber optic architectures for strain monitoring of solid rocket motors' propellant." Sens. Lett., 11(8), 1403-1407.

6. Lopatin, C., and Grinstein, D. (2015). "Active sensing for monitoring the properties of solid rocket motor propellant grains." Propellants, Explos., Pyrotech., 40(2), 295-302.

7. Udd, E., and Benterou, J. (2012). "Improvements to high-speed monitoring of events in extreme environments using fiber Bragg grating sensors." Proc., SPIE: Fiber Optic Applications and Sensors IX, SPIE, Bellingham, WA, Vol. 8370, 83700L-183700L-13. 